\title{
Protein and Crystallography Facility at the University of Iowa
}

\author{
Zhen $\mathrm{Xu}^{1}$, Nicholas Schnicker ${ }^{1}$, Sankar Baruah ${ }^{1}$, Paul Rogers ${ }^{1}$, Irini Petros ${ }^{1}$, Lokesh \\ Gakhar $^{1,2}$ \\ ${ }^{1}$ Protein and Crystallography Facility, Roy J. and Lucille A. Carver College of Medicine, \\ University of lowa, lowa City, IA 52242 \\ ${ }^{2}$ Department of Biochemistry, Roy J. and Lucille A. Carver College of Medicine, University of \\ lowa, lowa City, IA 52242 \\ pcf@healthcare.uiowa.edu
}

The Protein and Crystallography Facility (PCF) at the University of lowa was officially established in 2004. It is an integrated structural biology and biophysics core facility which offers a wide variety of training, consulting, contract and technical support services including protein expression, purification, characterization, crystallization, X-ray diffraction data collection, data analysis etc. We have in-house state-of-art biolayer interferometry (BLI), dynamic/static lighting scattering (DLS/SLS), differential scanning fluorimetry (DSF), protein purification, crystallization, liquid handling and X-ray instrumentation. We have routine remote access to beamline 4.2.2 at the Advanced Light Source of Berkeley National Lab through consortium membership that operates the beamline.

In addition to X-ray crystallography to obtain high resolution structural information, we also use small-angle X-ray scattering (SAXS) integrated with other experimental and computational methods that rapidly provide a more thorough characterization of shapes, conformations, and assembly states of proteins in near physiological environments. We routinely use the size exclusion chromatography (SEC) in-line with multi-angle light scattering (MALS) and SAXS format of data collection at the Advanced Photon Source's 18-ID-D Bio-CAT beamline (Argonne National Lab).

Our mission is to provide basic science and drug discovery support to projects including, but not limited to, structural/functional studies of proteins, protein assemblies, protein/DNA complexes to academia and industry. The support includes providing expertise in experimental design, data collection, processing, analysis and interpretation.

This poster will introduce the Protein and Crystallography Facility at the University of lowa and present highlights of recent scientific achievements of our facility users.

Protein and Crystallography Facility website: https://medicine.uiowa.edu/crystallography/ 